Research Article

\title{
Pull-In and Snap-Through Analysis of Electrically Actuated Viscoelastic Curved Microbeam
}

\author{
Ehsan Akrami-Nia (iD and Hamid Ekhteraei-Toussi (i) \\ Department of Mechanical Engineering, Ferdowsi University of Mashhad, Mashhad 9177948974-1111, Iran \\ Correspondence should be addressed to Hamid Ekhteraei-Toussi; ekhteraee@um.ac.ir
}

Received 7 March 2020; Revised 24 May 2020; Accepted 1 June 2020; Published 9 July 2020

Academic Editor: Jose Cesar de Sa

Copyright (c) 2020 Ehsan Akrami-Nia and Hamid Ekhteraei-Toussi. This is an open access article distributed under the Creative Commons Attribution License, which permits unrestricted use, distribution, and reproduction in any medium, provided the original work is properly cited.

\begin{abstract}
Microbeams are key elements in most of the micro-electromechanical systems (MEMS). Electromechanical instability of microbeams in turn plays an important role in their applications. The shape and mechanical properties of microbeams dictate their functional characteristics. Focusing on their instability-based working mechanism, one can appreciate that viscoelasticity of MEMS materials cannot be neglected. Consequently, the analysis of instability in viscoelastic curved microbeams is an essential demand. In this research, assuming a clamped-clamped initially curved microbeam, the effects of viscoelastic behavior on the snap-through and pull-in instabilities are investigated. The standard inelastic linear solid model is used for the simulation of viscoelastic behavior. Integrodifferential governing equation of the curved viscoelastic microbeam is obtained by assuming modified couple stress theory and using Hamilton's principle. By applying the Galerkin method, the obtained governing equation is discretized, converted to a nonlinear differential equation, and solved by MATLAB software. Through a quasi-static analysis, the voltage and location of snap-through and pull-in instabilities are identified. The effects of different viscoelastic parameters including the creep moduli and relaxation coefficient upon the snap-through and pull-in instabilities are investigated. The effects of different short- and long-term creeping characteristics of viscoelastic microbeam are studied and discussed in detail.
\end{abstract}

\section{Introduction}

Micro-electromechanical systems (MEMS) are widely used in modern daily life and more sophisticated technical devices such as the cellphones or car-making industries. Due to their unique advantages such as less power consumption and smaller size/weight compared to traditional mechanical systems, the utilization of these systems is developed in different industries. MEMS are increasingly identified as one of the most promising technologies for the twenty-first century $[1,2]$. The various aspects of economy and social development, ranging from automotive and medical to electronic, communication, and defense applications represent widespread use of these devices. With their great potential to revolutionize the industry and upgrade various products, MEMS are attracting global scientific efforts and funding. Over the last three decades, global investment in this area has increased dramatically. According to 2014-Yole
Développement Report, it is projected that global investment in this promising area is above 20 billion USD by 2020 [3].

Because of their geometric simplicity, broad applicability, and easy to implement characteristic, microbeams are perhaps the most commonly used structural component in MEMS. These components form the backbone of a wide range of devices including the resonators, resonant sensors, actuators, filters, atomic force microscope probes, and RF switches [4]. Because of their special role in MEMS and their different actuation methods such as mechanical, electrical, thermal, and magnetic fields, microbeams have made a newly research frontier. These devices are typically actuated by various actuation methods such as thermal, piezoelectric, or electrostatic stimuli. The electrically actuated MEMS devices which require few mechanical components, small voltage levels for actuation, and lower power consumption to combine with microprocessors are continuously growing 
[5]. Therefore, studying the behavior and controlling the deflection of electrical microbeams is an important demand. Microbeams are liable to a kind of instability usually known as pull-in effect. This kind of instability is a result of interaction in between the elastic and electrostatic forces produced by applied voltage. When the voltage increases beyond a critical value, called the pull-in voltage, elastic force can no longer resist electrostatic force. This condition leads to a rapid escape of microbeam to contact the electrode. This instability was first shown empirically by Taylor [6] and Nathanson et al. [7] and named as "Pull-in" instability. Therefore, in terms of sustainability, these systems are considered as vulnerable structures.

In order to control and improve the performance of MEMS devices, various methods are presented. One of the most widely used mechanisms are curved structures. These types of beams have attracted increasing interests in the research community in recent years. Here, curved beam refers to a beam fabricated intentionally in curved shape or made curved by buckling straight beams through compressive axial loads [8]. Recent works have shown that depending on the interaction between electrical and mechanical forces, these structures can show favorable features. One of these behaviors is the large deflection jump in between the two stable states, which is called the snap-through phenomenon. Qiu et al. [9] are among the first who recognized this phenomenon and introduced these structures as bistable systems. Their bistable mechanism comprises two initially sine-shaped beams clamped together at the center that have no residual stress after fabrication. The bistability nature is one of the major advantages of the curved structures which makes them suitable for actuation and switching applications. In particular, they do not require power to be kept in each one of their stable states, even if they need power to change their stable states. The mechanical behaviors of MEMS arches, in particular their pullin and snap-through instabilities, are widely studied to explore static and dynamic characteristics of these systems. Early studies mainly focused on the static and dynamic behavior of elastic curved microbeam which assumes constant elastic modulus. For example, using constant elastic modulus, Zhang et al. $[10,11]$ investigated snap-through and pull-in instabilities of micromachined arch-shaped beam under electrostatic loading. Their results predict that probability of pull-in or snap-through is depended on the size of microbeam, its curvature, and the level of electrostatic loading. Their simulation model is based on Euler-Bernoulli thin beam and Galerkin method. Ouakad and Younis [8] studied the static and dynamic behaviors of clampedclamped micromachined arches actuated by a small DC electrostatic load superimposed to an AC harmonic load. They calculated the natural frequencies and mode shapes of the arch for various values of DC voltages and initial rises. Their results indicated that resonance frequency is significantly lowered as the values of DC and AC loads are increased and the values of initial rise are decreased. The dynamic pull-in instability and snap-through buckling of initially curved microbeams due to a suddenly applied voltage have been investigated by Zand [12]. He considered the effects of midplane stretching load and employed a finite element model and Newmark time discretization to solve the equations. He found that, by increasing the initial elevation, the pull-in voltage decreases while the snap-through voltage increases. His results indicated that a qualitative change in the character of microbeam response is observed when the voltage parameter exceeds snap-through or pull-in values. It was noticed that, for some levels of initial elevation, the beam would jump to contact rigid electrode immediately after snap-through incidence. It was also shown that inertia of the beam has more influence on the snap-through than on pullin instability. Results of his study are beneficial in MEMS applications including mechanical memories and optical switches.

Although all previous researches about curved microbeams are based on the assumption of pure elasticity $[8,12,13]$, some recent experimental results have shown that viscoelastic behavior widely exists in many materials used in the construction of MEMS devices such as silicon [14, 15], polysilicon [16-18], and metals [19, 20]. Therefore, the consideration of viscous dissipative force in microbeams seems to be necessary. In this regard, Fu and Zhang [21] investigated nonlinear static and dynamic responses of a viscoelastic clamped-clamped microbeam under a purely DC polarization voltage. They have shown that once a viscoelastic microbeam is under purely DC voltage, not only may an instantaneous pull-in instability have happened, but also a durable type of pull-in instability resulting from the creeping effect of viscoelastic material might have sensed. The simulation of viscoelastic behavior of microbeams has been carried out according to various material models, such as Kelvin-Voigt model (by Ghayesh et al. [22]) and Maxwell model (by Attia and Mohamed [23]). Li et al. [24] investigated the static and dynamic behaviors of a doubly clamped viscoelastic straight microbeam actuated by one sided electrode. Their simulations were based on the standard inelastic linear solid model for the material viscoelasticity and modified couple stress theory. Through quasi-static analysis, they obtained pull-in voltage and pull-in location of the system with differential quadrature and finite element methods. Similar to Fu and Zhang [21], these researchers also predicted two types of instantaneous and durable pullin voltage for the viscoelastic structure. Many other researchers showed that different from elastic material, there are two kinds of instantaneous and durable instabilities in viscoelastic system. But previous research studies either studied viscoelastic straight beams or considered nonviscoelastic (i.e., elastic) curved beams. The beam models used in the curved systems mainly belong to the classical elastic theory. The snap-through and pull-in instabilities are studied with this assumption. The same model of beam theory can be used to study instabilities of viscoelastic microbeam. Accordingly, in the present study, assuming a clamped-clamped initially curved microbeam, the effects of viscoelastic behavior on the snap-through and pull-in instabilities have been investigated. The viscoelastic behavior is modeled by standard an-elastic linear solid model. Using modified couple stress theory and Hamilton's principle, the governing integrodifferential equation is obtained. The 
resulting equation is discretized and converted to a nonlinear integrodifferential equation by using the reducedorder model known as Galerkin's procedure. The resulting equation is solved by MATLAB software and microbeam response is obtained. Performing a quasi-static analysis and considering the variation of maximum deflection versus the voltage, the location and voltage of snap-through and pull-in instabilities are calculated for different values of creep moduli and relaxation coefficient. Finally, the effects of different material parameters upon these instabilities are studied. So, the main novelties sought in this paper would be the extraction and solution of integrodifferential equations of curved viscoelastic microbeam as well as the introduction of snap-through and pull-in phenomena in instantaneous and durable states under the effect of creep modulus and relaxation coefficient of element.

\section{Materials and Methods}

In this section, the nonlinear integrodifferential governing equation and boundary conditions of an actuated viscoelastic curved microbeam subjected to electrical loads, midplane stretching, and residual stress effects are extracted. Figure 1 shows a MEMS arch resonator in which deformable electrode is modeled by initially curved electrically actuated microbeam of length $L$, width $b$, thickness $h$, and gap-width $d$. The initial curvature of the microbeam is denoted by $w_{0}(x)$.

2.1. Problem Formulation. As shown in Figure 1, the initial curvature of microbeam $w_{0}(x)$ is extended outside of the gap. The transverse displacement of microbeam happening in $Z$-direction is denoted by $w(x, t)$. An electrical load actuation is applied to deformable electrode in transversal direction. In this work, it is assumed that, initially, the deformable electrode deserves a slightly curved shape, that is, $\partial w_{0}(x) / \partial x \ll 1$ and one can use parallel-plate assumption to determine electrical force. Based on the selected assumptions of curvature and $Z$-axis direction in Figure 1, initial curvature of the microbeam is defined as follows [25]:

$$
\widehat{w}_{0}(x)=-\frac{d_{0}}{2}\left(1-\cos \left(\frac{2 \pi \widehat{x}}{L}\right)\right)
$$

where $d_{0}$ is the maximum initial curvature of the microbeam.

The standard an-elastic linear solid model which can be reasoned from most experimental results such as $[21,24]$ is introduced for the material viscoelasticity and the relaxation function is given as

$$
\widehat{E}(t)=\widehat{Z}+\widehat{B} e^{-\widehat{\lambda} t},
$$

where $\widehat{\lambda}$ is the element relaxation coefficient of MEMS material and $\widehat{Z}$ and $\widehat{B}$ are, respectively, the durable and creep moduli of viscoelastic microbeam. It should be noted that when a wide microbeam $(b \geq 5 h)$ is considered, the relaxation function should be changed to $\widehat{E}(t) /\left(1-v^{2}\right)$, where $v$ denotes Poisson's ratio which is time independent. Equation (2) implies an initial Young's modulus of $\widehat{E}_{0}=\widehat{E}(0)=\widehat{Z}+\widehat{B}$. Taking into account

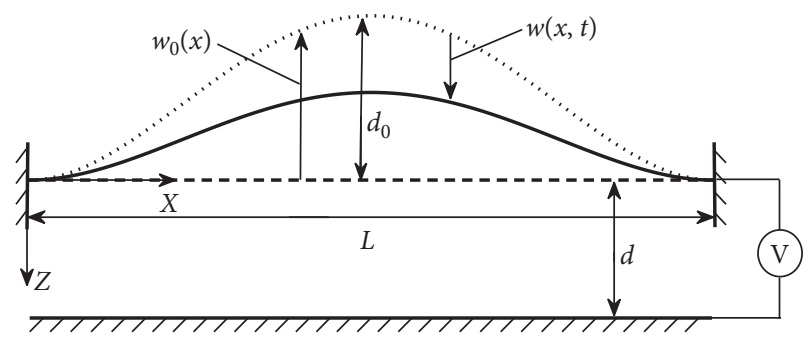

FIGURE 1: Schematic of a clamped-clamped electrically actuated initially curved microbeam.

the midplane stretching effect and neglecting the Casimir and Van der Waals forces, the governing equation of viscoelastic straight microbeam in terms of transverse deflection $\widehat{w}(\widehat{x}, t)$ is obtained by Li et al. [24]. This formulation relies on Hamilton's principle and the theory of modified couple stress:

$$
\begin{aligned}
& \left(\widehat{E}_{0} I+\widehat{\mu}_{0} A l^{2}\right) \frac{\partial^{4} \widehat{w}(\widehat{x}, t)}{\partial \widehat{x}^{4}}+\rho A \frac{\partial^{2} \widehat{w}(\widehat{x}, t)}{\partial t^{2}}+\int_{0}^{t}\left(\frac{\partial \widehat{E}\left(t-t_{0}\right)}{\partial\left(t-t_{0}\right)} I\right. \\
& \left.+\frac{\partial \widehat{\mu}\left(t-t_{0}\right)}{\partial\left(t-t_{0}\right)} A l^{2}\right) \frac{\partial^{4} \widehat{w}(\widehat{x}, t)}{\partial \widehat{x}^{4}} \mathrm{~d} t_{0} \\
& =\left\{\widehat{P}_{r}+\frac{\widehat{E}_{0} A}{2 L} \int_{0}^{L}\left(\frac{\partial \widehat{w}(\widehat{x}, t)}{\partial \widehat{x}}\right)^{2} \mathrm{~d} \widehat{x}+\frac{A}{2 L} \int_{0}^{t}\left(\frac{\partial \widehat{E}\left(t-t_{0}\right)}{\left(t-t_{0}\right)}\right.\right. \\
& \left.\left.\int_{0}^{L}\left(\frac{\partial \widehat{w}(\widehat{x}, t)}{\partial \widehat{x}}\right)^{2} \mathrm{~d} \widehat{x}\right) \mathrm{~d} t_{0}\right\} \frac{\partial^{2} \widehat{w}(\widehat{x}, t)}{\partial \widehat{x}^{2}}+\frac{\varepsilon b V^{2}(t)}{2(d-\widehat{w}(\widehat{x}, t))^{2}} .
\end{aligned}
$$

The axial load and midplane stretching effects are represented in the first term on the right side of (3) and the influence of relaxation effect is introduced via the integrated terms on both sides of the equation. In this relation, $\widehat{E}(t)$ and $\widehat{\mu}(t)$ are time depended Young's modulus and Lame's constant while $\widehat{E}_{0}$ and $\widehat{\mu}_{0}$ are the initial Young's modulus and Lame's constant. The symbols $A$ and $I$ are the area and moment of inertia of the beam cross section. $L$ is a material length scale parameter. Moreover, $\hat{x}$ is the position along the microbeam length. $t$ is time. $\rho$ is material density. $L$ is the length of microbeam. $d$ is the distance between the direction of the microbeam supports and the electrode which is the same as the gap-width for the straight microbeam. $\varepsilon$ is the dielectric constant of the gap medium and $V(t)$ is the applied voltage. The parameter $\widehat{P}_{r}$ stands for the tensile or compressive axial component of load. The last term in (3) indicates the electrical force and depends on both the voltage and position of microbeam. Therefore, both voltage and position levels would jointly affect the stability of the system.

The boundary conditions for the clamped-clamped end conditions are expressed as

$$
\widehat{w}(0, t)=\frac{\partial \widehat{w}(0, t)}{\partial \widehat{x}}=\widehat{w}(L, t)=\frac{\partial \widehat{w}(L, t)}{\partial \widehat{x}}=0
$$

As shown in Figure 1, if the straight microbeam is replaced by a curved microbeam, the deflection of 
microbeam must be replaced by $\widehat{w}(\widehat{x}, t)+\widehat{w}_{0}(\widehat{x})$. But, the terms on the left side of equation (3) that represent kinetic energy and strain energy depend on $\widehat{w}(\widehat{x}, t)$. By expansion of substituted terms and using thin beam assumption, the effect of $\left[\mathrm{d} \widehat{w}_{0}(\hat{x}) / \mathrm{d} \hat{x}\right]^{2}$ is reduced and neglected. Thus, the governing equation of transversal deflection $\widehat{w}(\widehat{x}, t)$ of viscoelastic curved microbeam is written as

$$
\begin{aligned}
\left(\widehat{E}_{0} I\right. & \left.+\widehat{\mu}_{0} A l^{2}\right) \frac{\partial^{4} \widehat{w}(\widehat{x}, t)}{\partial \widehat{x}^{4}}+\rho A \frac{\partial^{2} \widehat{w}(\widehat{x}, t)}{\partial t^{2}}+\int_{o}^{t}\left(\frac{\partial \widehat{E}\left(t-t_{0}\right)}{\partial\left(t-t_{0}\right)} I+\frac{\partial \widehat{\mu}\left(t-t_{0}\right)}{\partial\left(t-t_{0}\right)} A l^{2}\right) \frac{\partial^{4} \widehat{w}\left(\widehat{x}, t_{0}\right)}{\partial \widehat{x}^{4}} \mathrm{~d} t_{0} \\
= & \left\{\widehat{P}_{r}+\frac{\widehat{E}_{0} A}{2 L} \int_{0}^{L}\left[\left(\frac{\partial \widehat{w}(\widehat{x}, t)}{\partial \widehat{x}}\right)^{2}+2 \frac{\partial \widehat{w}(\widehat{x}, t)}{\partial \widehat{x}} \frac{\mathrm{d} \widehat{w}_{0}(\widehat{x})}{\mathrm{d} \widehat{x}}\right] \mathrm{d} \widehat{x}+\frac{A}{2 L} \int_{0}^{t}\left(\frac{\partial \widehat{E}\left(t-t_{0}\right)}{\partial\left(t-t_{0}\right)} \int_{0}^{L}\left[\left(\frac{\partial \widehat{w}\left(\widehat{x}, t_{0}\right)}{\partial \widehat{x}}\right)^{2}+2 \frac{\partial \widehat{w}\left(\widehat{x}, t_{0}\right)}{\partial \widehat{x}} \frac{\mathrm{d} \widehat{w}_{0}(\widehat{x})}{\mathrm{d} \widehat{x}}\right] \mathrm{d} \widehat{x}\right) \mathrm{d} t_{0}\right\} \\
& \cdot\left[\frac{\partial^{2} \widehat{w}(\widehat{x}, t)}{\partial \widehat{x}^{2}}+\frac{\mathrm{d}^{2} \widehat{w}_{0}(\widehat{x})}{\mathrm{d} \widehat{x}^{2}}\right]+\frac{\varepsilon V^{2}(t)}{2\left(d-\widehat{w}_{0}(\widehat{x})-\widehat{w}(\widehat{x}, t)\right)^{2}}
\end{aligned}
$$

For convenience, the following nondimensional variables are defined:

$$
\begin{aligned}
& w=\frac{\widehat{w}}{d+d_{0}}, \\
& x=\frac{\widehat{x}}{L}, \\
& \tau=\frac{t}{T},
\end{aligned}
$$

$$
\begin{aligned}
& \tau_{0}=\frac{t_{0}}{T} \\
& T=\sqrt{\frac{\rho A L^{4}}{E_{0} I}} .
\end{aligned}
$$

Substituting the nondimensional variables into equations (4) and (5) and dropping the hat for simplicity, the following nondimensional equation of motion is obtained:

$$
\begin{aligned}
& \frac{\partial^{2} w(x, \tau)}{\partial \tau^{2}}+(1+\eta)\left[\frac{\partial^{4} w(x, \tau)}{\partial x^{4}}+\int_{0}^{\tau} \frac{\partial n\left(\tau-\tau_{0}\right)}{\partial\left(\tau-\tau_{0}\right)} \frac{\partial^{4} w\left(x, \tau_{0}\right)}{\partial x^{4}} \mathrm{~d} \tau_{0}\right] \\
& =\left\{P_{r}+\alpha \int_{0}^{1}\left[\left(\frac{\partial w(x, \tau)}{\partial x}\right)^{2}+2 \frac{\partial w(x, \tau)}{\partial x} \frac{\mathrm{d} w_{0}(x)}{\mathrm{d} x}\right] \mathrm{d} x+\alpha \int_{0}^{\tau}\left(\frac{\partial n\left(\tau-\tau_{0}\right)}{\partial\left(\tau-\tau_{0}\right)} \int_{0}^{1}\left[\left(\frac{\partial w\left(x, \tau_{0}\right)}{\partial x}\right)^{2}+2 \frac{\partial w\left(x, \tau_{0}\right)}{\partial x} \frac{\mathrm{d} w_{0}(x)}{\mathrm{d} x}\right] \mathrm{d} x\right) \mathrm{d} \tau_{0}\right\} \\
& \quad \cdot\left[\frac{\partial^{2} w(x, \tau)}{\partial x^{2}}+\frac{\mathrm{d}^{2} w_{0}(x)}{\mathrm{d} x^{2}}\right]+\frac{\beta V^{2}(\tau)}{\left(1-w_{0}(x)-w(x, \tau)\right)^{2}} .
\end{aligned}
$$

Its boundary conditions are

$$
w(0, \tau)=\frac{\partial w(0, \tau)}{\partial x}=w(1, \tau)=\frac{\partial w(1, \tau)}{\partial x}=0 .
$$

The nondimensional parameters used in equation (7) are presented in Table 1.

2.2. Reduced-Order Model. Due to the high nonlinearity involved in equation (7), a closed-form solution for this equation cannot be found. Hence, an approximate solution based on Galerkin weighted residual method is developed [26]. To this end, the deflection is expressed as

$$
w(x, \tau)=\sum_{i=1}^{N} \varphi_{i}(x) u_{i}(\tau)
$$

where $N$ is the number of degrees of freedom, $u_{i}(\tau)$ is $i$ th generalized coordinate, and $\varphi_{i}(x)$ is $i$ th nondimensional linear undamped mode shape of undeformed microbeam. 
TABle 1: Nondimensional parameter definitions.

\begin{tabular}{lc}
\hline Parameter & Definition \\
\hline$w_{0}=\widehat{w}_{0} /\left(d+d_{0}\right)$ & Initial curvature \\
$P_{r}=\widehat{P}_{r} L^{2} /\left(\widehat{E}_{0} I\right)$ & Axial load \\
$Z=\widehat{Z} / \widehat{E}_{0}$ & Durable modulus \\
$B=\widehat{B} / \widehat{E}_{0}$ & Creep modulus \\
$\lambda=\widehat{\lambda} T$ & Relaxation coefficient \\
$\alpha=6(d / h)^{2}$ & Stretching parameter \\
$\beta=6 \varepsilon L^{4} /\left(\widehat{E}_{0} h^{3} d^{3}\right)$ & Electrical force parameter \\
$n(\tau)=Z+B e^{-\lambda \tau}$ & Relaxation function \\
$\eta=\widehat{\mu}_{0} A l^{2} /\left(\widehat{E}_{0} I\right)$ & Material length scale parameter \\
\hline
\end{tabular}

Now, $\varphi_{i}(x)$ is normalized such that $\int_{0}^{1} \varphi_{i}(x) \varphi_{j}(x) \mathrm{d} x=\delta_{i j}$, where $\delta_{i j}$ is the Kronecker delta. Since, in vibration analysis, the first mode is dominant $[26,27]$, the one-mode approximation has been widely used in the previous studies [27-29]. Henceforth, the deflection of microbeam based on one-mode solution can be expressed as

$$
w(x, \tau)=\varphi(x) u(\tau),
$$

where $u(\tau)$ is unknown time-dependent deflection parameter and $\varphi(x)$ is the first linear and undamped mode shape of undeformed microbeam determined as $[8,24]$

$$
\begin{aligned}
\varphi(x)= & \cosh (4.73 x)-\cos (4.73 x)-0.9825[\sinh (4.73 x) \\
& -\sin (4.73 x)] .
\end{aligned}
$$

For convenience, the deflection of the microbeam can be expressed as

$$
w(x, \tau)=\psi(x) f(\tau)
$$

in which

$$
\psi(x)=\frac{\varphi(x)}{\varphi(0.5)} .
$$

Accordingly, in equation (12), $f(\tau)$ represents the maximum deflection of the microbeam midspan. Substituting equations (12) and (13) into equation (7) results in

$$
\begin{aligned}
(1+\eta) & {\left[f(\tau) \frac{\mathrm{d}^{4} \psi(x)}{\mathrm{d} x^{4}}-B \lambda \int_{0}^{\tau} e^{-\lambda\left(\tau-\tau_{0}\right)} f\left(\tau_{0}\right) \frac{\mathrm{d}^{4} \psi(x)}{\mathrm{d} x^{4}} \mathrm{~d} \tau_{0}\right]+\psi(x) \frac{\mathrm{d}^{2} f(\tau)}{\mathrm{d} \tau^{2}} } \\
= & \left\{P_{r}+\alpha \int_{0}^{1}\left[\left(\frac{\mathrm{d} \psi(x)}{\mathrm{d} x}\right)^{2} f^{2}(\tau)+2 f(\tau) \frac{\mathrm{d} \psi(x)}{\mathrm{d} x} \frac{\mathrm{d} w_{0}(x)}{\mathrm{d} x}\right] \mathrm{d} x-\alpha B \lambda \int_{0}^{\tau}\left(e^{-\lambda\left(\tau-\tau_{0}\right)} \int_{0}^{1}\left[\left(\frac{\mathrm{d} \psi(x)}{\mathrm{d} x}\right)^{2} f^{2}\left(\tau_{0}\right)+2 f\left(\tau_{0}\right) \frac{\mathrm{d} \psi(x)}{\mathrm{d} x} \frac{\mathrm{d} w_{0}(x)}{\mathrm{d} x}\right] \mathrm{d} x\right) \mathrm{d} \tau_{0}\right\} \\
& \cdot\left[f(\tau) \frac{\mathrm{d}^{2} \psi(x)}{\mathrm{d} x^{2}}+\frac{\mathrm{d}^{2} w_{0}(x)}{\mathrm{d} x^{2}}\right]+\beta \frac{V^{2}(\tau)}{\left(1-w_{0}(x)-f(\tau) \psi(x)\right)^{2}} .
\end{aligned}
$$

In order to simplify the computations and minimize the error of the approximate solution, we multiply both sides of equation (14) by $\left\{1-w_{0}(x)-f(\tau) \psi(x)\right\}^{2}$ and next multiply both sides of the resulting equation by $\psi(x)$ and integrate the outcome from $x=0$ to 1 . Finally, it yields the following integrodifferential equation in terms of the modal coordinate $f(\tau)$ :

$$
\begin{aligned}
& (1+\eta)\left[c_{1}-2 c_{2} f(\tau)+c_{3} f^{2}(\tau)-2 f_{1}+2 f_{2} f(\tau)+g\right] \\
& \left\{f(\tau)-B \lambda \int_{0}^{\tau} e^{-\lambda\left(\tau-\tau_{0}\right)} f\left(\tau_{0}\right) \mathrm{d} \tau_{0}\right\}+\left[a_{2}-2 a_{3} f(\tau)+a_{4} f^{2}(\tau)-2 h_{1}+h_{2}+2 m f(\tau)\right] \\
& \frac{\mathrm{d}^{2} f(\tau)}{\mathrm{d} \tau^{2}}=\left\{b_{1} f(\tau)-2 b_{2} f^{2}(\tau)+b_{3} f^{3}(\tau)+d_{1}-2 d_{2} f(\tau)+d_{3} f^{2}(\tau)-2 e_{1} f(\tau)+2 e_{2} f^{2}(\tau)-2 k_{1}+2 k_{2} f(\tau)+p f(\tau)+q\right\} \\
& {\left[P_{r}+\alpha r f^{2}(\tau)+2 \alpha s f(\tau)-\alpha B \lambda r \int_{0}^{\tau} e^{-\lambda\left(\tau-\tau_{0}\right)} f^{2}\left(\tau_{0}\right) \mathrm{d} \tau_{0}-2 \alpha B \lambda s \int_{0}^{\tau} e^{-\lambda\left(\tau-\tau_{0}\right)} f\left(\tau_{0}\right) \mathrm{d} \tau_{0}\right]+\beta a_{1}^{2}(\tau) .}
\end{aligned}
$$

The definitions of constant factors in equation (15) are provided at the end of the paper.

By solving equation (15), the relationship between $f(\tau)$ and voltage is obtained. Hence, the stable or unstable behaviour of the system can be predicted, precisely.
Consequently, if the rate of maximum deflection change to voltage goes to infinity, an unstable situation will occur. In other words, after this point, microbeam does not need the increase of voltage to get closer to the electrode and collapse onto it. 
Here, a system with the geometrical and mechanical properties provided in Table 2 is selected to study the effect of viscoelastic behavior on the snap-through and pull-in instabilities in curved MEMS beams.

The parameters in Table 2 imitate the material properties of silicon as the basic constructive material in microelectromechanical systems $[5,8]$. Furthermore, the effective range of the parameters is selected according to the results provided in other studies on viscoelastic noncurved microbeams such as Zhang and Fu [5], Fu and Zhang [21], and Lee et al. [24].

2.3. Quasi-Static Analysis. Quasi-static analysis of MEMS provides the instability voltage and instability location of the system. By applying an increasing DC voltage to the structure, once the effect of inertia is negligible, the behavior of microstructure can be analyzed as a quasi-static problem. In this case, the time-depended section of the response is converted to a time-independent coefficient. Therefore, depending on the actuation voltage, the deflection of microbeam is introduced as
TABLE 2: Geometrical and mechanical properties of the viscoelastic curved system $[5,8]$.

\begin{tabular}{lc}
\hline Parameter & Quantity \\
\hline $\mathrm{L}$ & $1000 \mu \mathrm{m}$ \\
$b$ & $30 \mu \mathrm{m}$ \\
$h$ & $2.4 \mu \mathrm{m}$ \\
$d$ & $10.1 \mu \mathrm{m}$ \\
$d_{0}$ & $3.5 \mu \mathrm{m}$ \\
$\widehat{E}_{0}$ & $166 \mathrm{GPa}$ \\
$\lambda$ & 0.1 \\
$v$ & 0.22 \\
$B$ & 0.4 \\
$L$ & $0.15 \mu \mathrm{m}^{-3}$ \\
$\rho$ & $2332 \mathrm{kgm}^{-3}$ \\
\hline
\end{tabular}

$$
w(x)=a \psi(x)
$$

By setting all time derivatives in equation (15) equal to zero and substituting equation (16) into the resulting equation, static equilibrium equation is achieved as follows:

$$
\begin{aligned}
(1+\eta) & {\left[c_{1}-2 c_{2} a+c_{3} a^{2}-2 f_{1}+2 f_{2} a+g\right]\left\{a-B \lambda \int_{0}^{\tau} e^{-\lambda\left(\tau-\tau_{0}\right)} a \mathrm{~d} \tau_{0}\right\} } \\
= & \left\{b_{1} a-2 b_{2} a^{2}+b_{3} a^{3}+d_{1}-2 d_{2} a+d_{3} a^{2}-2 e_{1} a+2 e_{2} a^{2}-2 k_{1}+2 k_{2} a+p a+q\right\} \\
& \cdot\left[P_{r}+\alpha r a^{2}+2 \alpha s a-\alpha B \lambda r \int_{0}^{\tau} e^{-\lambda\left(\tau-\tau_{0}\right)} a^{2} \mathrm{~d} \tau_{0}-2 \alpha B \lambda s \int_{0}^{\tau} e^{-\lambda\left(\tau-\tau_{0}\right)} a \mathrm{~d} \tau_{0}\right]+\beta a_{1} V^{2}(\tau) .
\end{aligned}
$$

The coefficient " $a$ " indicates the midpoint or maximum deflection of microbeam which is obtained for different voltages by solving equation (17). Plotting maximum displacement versus the voltage, one can determine the behaviour of the system including the location and voltage of instability. As noted earlier, an instable condition appears at the point where the microbeam deflection increases without any need for the rise of voltage. At this point, the rate of maximum deflection relative to applied voltage is infinite. In other words, the instability begins at the point that voltage gradient is deceased relative to the maximum deflection of microbeam. Mathematically, by using MATLAB <ezplot> command and equation (17), the amount of maximum deflection is plotted versus the potential difference. For this purpose, equation (17) results in the voltage-displacement correlation as follows:

$$
\begin{aligned}
V^{2}(\tau)= & \left(\frac{1}{\beta a_{1}}\right)\left\{(1+\eta)\left[c_{1}-2 c_{2} a+c_{3} a^{2}-2 f_{1}+2 f_{2} a+g\right]\left\{a-B \lambda \int_{0}^{\tau} e^{-\lambda\left(\tau-\tau_{0}\right)} a \mathrm{~d} \tau_{0}\right\}-\left\{b_{1} a-2 b_{2} a^{2}+b_{3} a^{3}+d_{1}\right.\right. \\
& \left.-2 d_{2} a+d_{3} a^{2}-2 e_{1} a+2 e_{2} a^{2}-2 k_{1}+2 k_{2} a+p a+q\right\} \cdot\left[P_{r}+\alpha r a^{2}+2 \alpha s a-\alpha B \lambda r \int_{0}^{\tau} e^{-\lambda\left(\tau-\tau_{0}\right)} a^{2} \mathrm{~d} \tau_{0}\right. \\
& \left.\left.-2 \alpha B \lambda s \int_{0}^{\tau} e^{-\lambda\left(\tau-\tau_{0}\right)} a \mathrm{~d} \tau_{0}\right]\right\} .
\end{aligned}
$$

In limit, the integrals in the second side of the equation (18) are definite. In instantaneous state $(\tau=0)$, these integrals are vanished and the terms containing them are removed. Therefore, in instantaneous state, the voltage and displacement relationship can be provided as in the following equation:. 


$$
\begin{aligned}
V^{2}(\tau)= & \left(\frac{1}{\beta a_{1}}\right)\left\{( 1 + \eta ) \left[c_{1} a-2 c_{2} a^{2}+c_{3} a^{3}-2 f_{1} a+2 f_{2} a^{2}\right.\right. \\
& +g a]-\left\{b_{1} a-2 b_{2} a^{2}+b_{3} a^{3}+d_{1}-2 d_{2} a+d_{3} a^{2}\right. \\
& \left.-2 e_{1} a+2 e_{2} a^{2}-2 k_{1}+2 k_{2} a+p a+q\right\} \\
& \left.\cdot\left[P_{r}+\alpha r a^{2}+2 \alpha s a\right]\right\} .
\end{aligned}
$$

In durable state $(\tau=\infty)$, one obtains

$$
\begin{aligned}
& \lim _{\tau \longrightarrow \infty} \int_{0}^{\tau} e^{-\lambda\left(\tau-\tau_{0}\right)} a \mathrm{~d} \tau_{0}=\frac{a}{\lambda}, \\
& \lim _{\tau \longrightarrow \infty} \int_{0}^{\tau} e^{-\lambda\left(\tau-\tau_{0}\right)} a^{2} \mathrm{~d} \tau_{0}=\frac{a^{2}}{\lambda} .
\end{aligned}
$$

Substituting equations (20) and (21) in equation (18), after some simplification, the voltage-displacement relationship for the durable state is obtained as follows:

$$
\begin{aligned}
V^{2}(\tau)= & \left(\frac{1}{\beta a_{1}}\right)\left\{(1+\eta) \hat{Z}\left[c_{1} a-2 c_{2} a^{2}+c_{3} a^{3}-2 f_{1} a+2 f_{2} a^{2}+g a\right]-\left\{b_{1} a-2 b_{2} a^{2}+b_{3} a^{3}+d_{1}-2 d_{2} a+d_{3} a^{2}-2 e_{1} a\right.\right. \\
& \left.\left.+2 e_{2} a^{2}-2 k_{1}+2 k_{2} a+p a+q\right\} \cdot\left[P_{r}+\alpha \widehat{Z} r a^{2}+2 \alpha \widehat{Z} s a\right]\right\} .
\end{aligned}
$$

Therefore, by using equations (19) and (22) for instantaneous and durable states and plotting the diagram of the voltage difference versus displacement by using MATLAB <ezplot> command, in each case, the trend of maximum displacement versus the voltage and, as a result, stability behavior of microbeam is specified. In each case by increasing the displacement level without any need to increase the voltage, instability would happen. In other words, zero changes in voltage versus displacement will indicate the onset of instability. Therefore, by solving the equations obtained by taking derivative of equations (19) and (22) with respect to displacement $(a)$ equal to zero, the configuration of system at the onset of instability is determined in instantaneous and durable states. The obtained equations are solved by <fzero $>$ command in MATLAB. Substituting the values obtained for the displacements back into equations (19) and (22), the starting voltage of snap-through and pullin corresponding to each of the states is determined. The solution method is implemented according to the following flowchart (Figure 2).

2.4. Validation. To validate the results, maximum deflections are compared with those reported in the literature. Nonetheless, the nonstraightness of beam and its viscoelastic behavior are two important contributions of the present work which has not been studied simultaneously in previous researches. Therefore, in order to validate the present model, results of simulation for initially curved microbeam and its viscoelastic behavior are compared with available literature, separately. To do this, primarily the maximum deflection of an elastic curved microbeam stimulated by a changing voltage is obtained by setting the creep modulus $B$ of the viscoelastic microbeam equal to zero. Figure 3 shows the results in comparison with the results of Ouakad and Younis [8].

The good agreement between the results in Figure 3 confirms that simulation is valid for the curved microbeams. Similarly, in order to validate the viscoelastic behavior, the results obtained for the viscoelastic straight microbeam are compared with the results of $\mathrm{Li}$ et al. [24]. For this purpose, the variation of maximum deflection for a viscoelastic straight microbeam described by $L=150 \mu \mathrm{m}, b=10 \mu \mathrm{m}$, $h=2 \mu \mathrm{m}, d=2 \mu \mathrm{m}, \eta=0.25$, and $d_{0}=0$ and mechanical properties $E_{0}=169 \mathrm{GPa}, \rho=2300 \mathrm{kgm}^{-3}, B=0.4$, and $v=0.22$ are obtained in its instantaneous and durable extremities. Figure 4 shows our results in comparison with the results of Li et al. [24].

A comparison between the stable behaviors stated in reference [24] and the results obtained in the present study shows good agreement between the solutions. Therefore, one can conclude that the simulation of viscoelasticity, the solution method, and the prediction of system instability are accurate and reliable.

\section{Results and Discussion}

Contrary to elastic material in which the mechanical behavior can be simplified by using lumped spring elements, the behavior of viscoelastic structure is simulated by combinations of lumped spring-damper components. Here the respond of damper element depends on the relaxed or selected time of study. If applied force is a constant value, the displacement change would continue during the time. In other word as shown in equation (7), the viscoelastic deflection of microbeam would depend on the assigned relaxation time, $\tau$. Therefore, in studies concerning viscoelastic behaviors, two types of instantaneous and durable responds can be studied. The instantaneous behavior is the response of structure immediately after the voltage actuation and is obtained by setting $\tau=0$ in the governing equation. In fact, this response reflects the purely elastic characteristic of the material behavior in which deformation occurs instantaneously in a moment and does not take any time trice. But the durable response means the maximum possible displacement of the structure, without having any time limitation. Therefore, durable response is long-term structure position obtained by setting $\tau=\infty$ in equilibrium equation. 


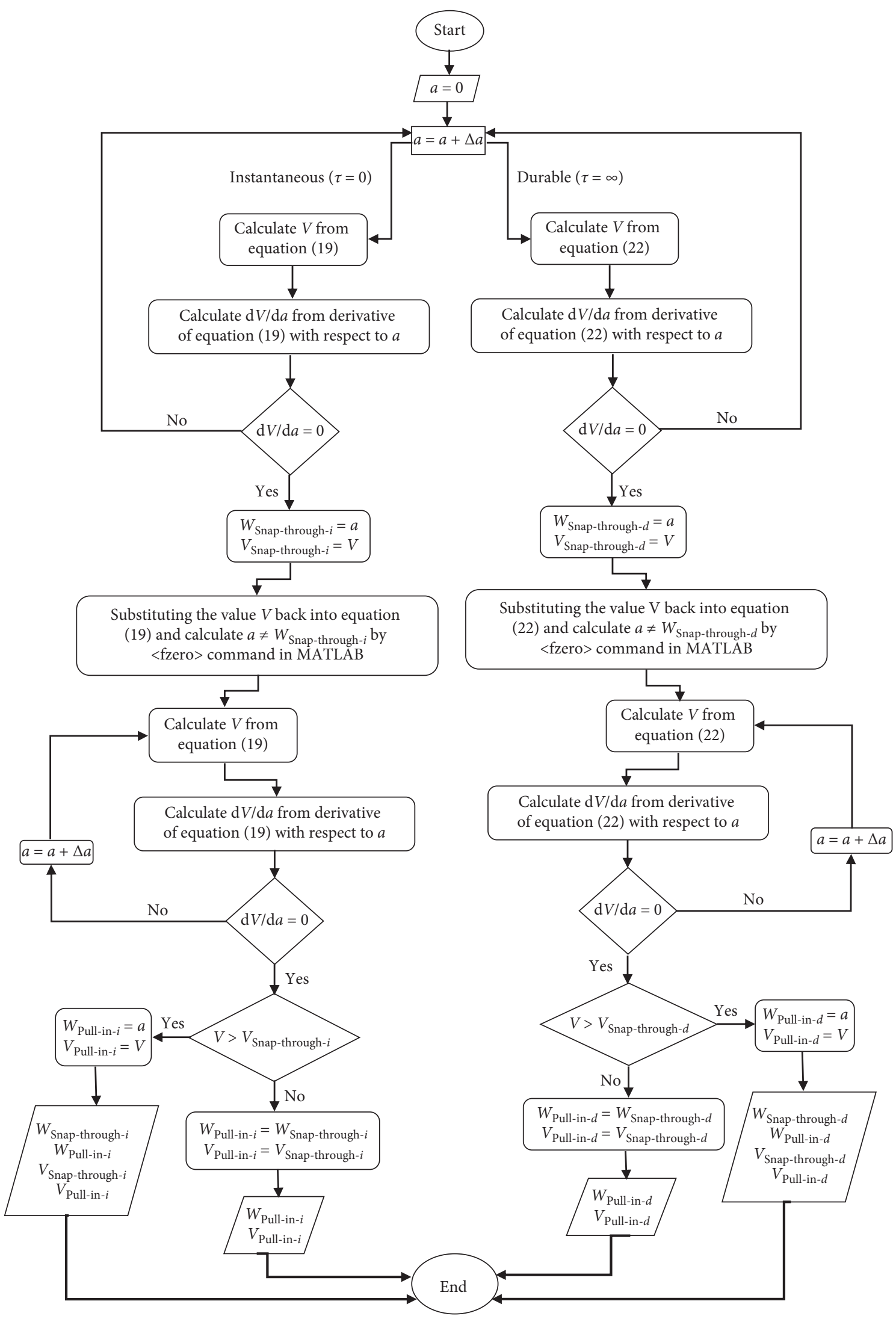

FIGURE 2: The algorithm of solution procedure. 


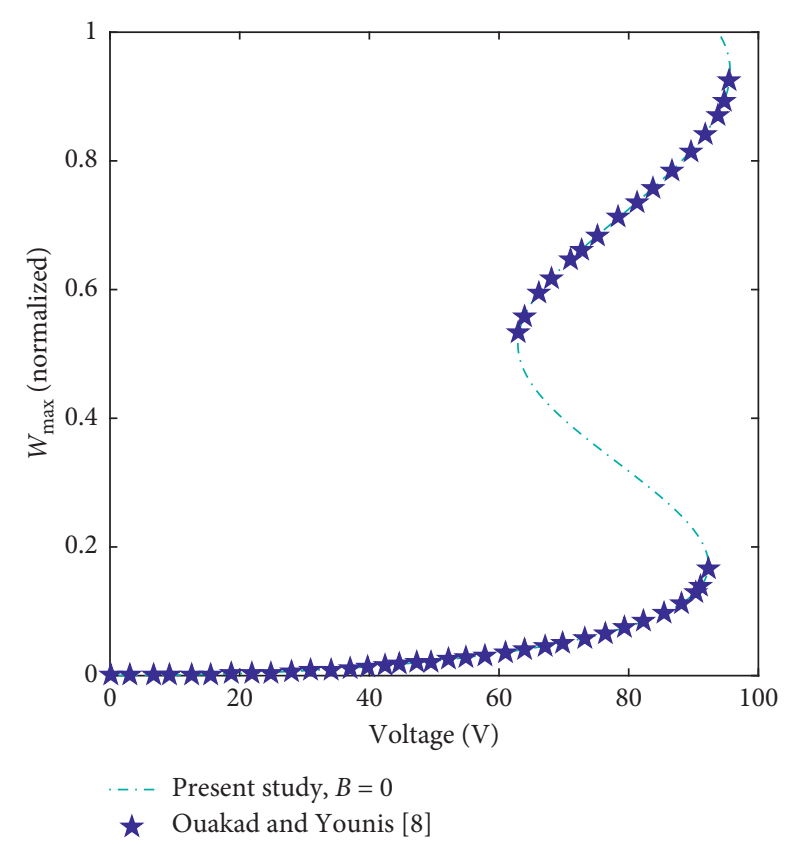

FIGURE 3: Maximum deflection of elastic curved microbeam versus the voltage.

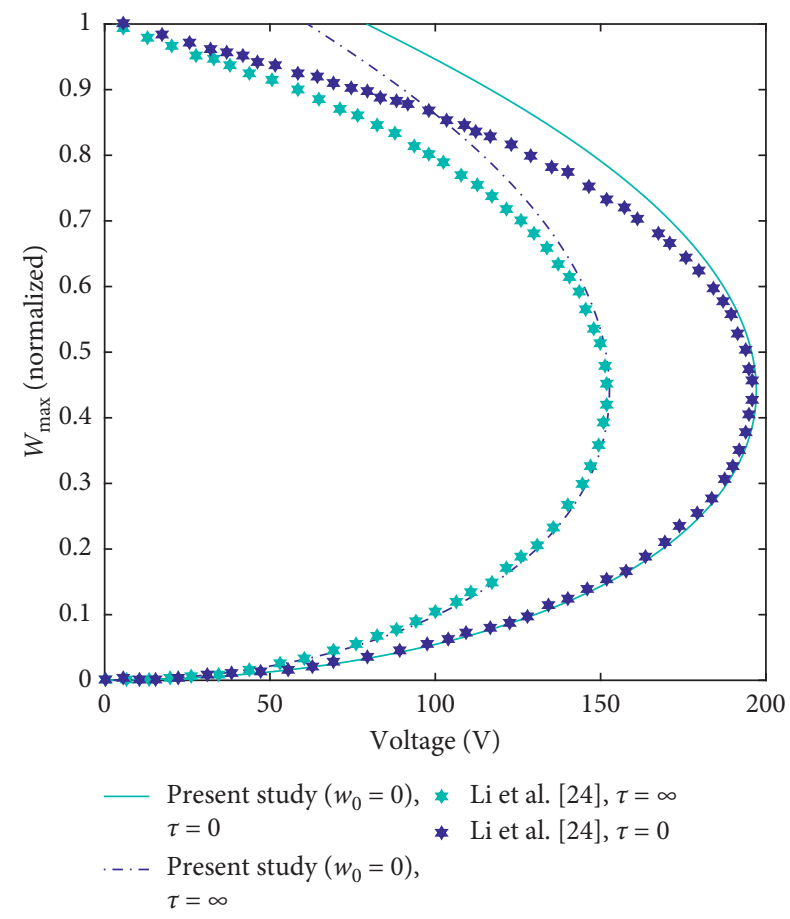

FIgURE 4: Maximum instantaneous and durable deflection of viscoelastic straight microbeam versus the voltage.

Thus, in order to investigate the effect of relaxation time upon the stability of viscoelastic curved microbeam, one can compare the structure behavior in these two extreme states. Consequently, from now on the position of structure is studied in two extreme conditions in different amounts of actuation voltage: first, immediately after the actuation instance and, next, after a long-time trice to achieve steady state condition. Figure 5 shows the results of this investigation for the system introduced in Table 2.

According to Figure 5, at any voltage level, structure achieves its durable stable position at larger displacement level comparing its instantaneous jump. This increase in the displacement of microbeam is such that durable position at a given voltage exceeds the instantaneous displacement of the structure at some larger voltages. For example, the stable position of a system with an operating voltage of $95 \mathrm{~V}$ is greater than the instantaneous response of a system with a voltage of $110 \mathrm{~V}$. This means that long-term effect of viscoelastic behavior is even greater than the effect of 15 -volt increase in voltage.

The durable state represents the viscoelastic behavior of material. As shown in Figure 5, creeping behavior can affect final position of the structure in three different forms. Either the behavior causes more displacement in the stable condition or transports the microbeam from its first quasi-stable state to another second stable state or even causes a jump from the second stable configuration into the pulled-in situation. The magnitude of the snap-through and pull-in voltages in instantaneous and durable states determines how viscoelastic behavior affects the function of microbeam. Since, the stability of the structure is determined by its maximum deflection, for the system with characteristics shown in Table 2, the variation of maximum transverse displacement of microbeam versus the voltage is obtained in two instantaneous and durable states. Figure 6 shows the variation of maximum deflection versus the voltage in these extreme states, in the absence of axial load.

In Figure 6, the solid line represents the stable solution and the pointed line introduces the system instability. Therefore, by increase of voltage, firstly the curved microbeam undergoes a snap-through which flips the microbeam into a new stable configuration. Then, by more increase of voltage, in a position closer to the fixed electrode, the microbeam becomes unstable and collapses onto the electrode. The snap-through causes the microbeam to nigh the electrode by changing its position between two stable states, without increasing the voltage. As a result, the curved microbeam has less voltage level in comparison to the straight microbeam in a position more near into the electrode. This feature helps to retain the curved microbeam stable in a range closer to the electrode in comparison with the straight microbeam. That is, snap-through phenomenon helps the system to use greater percentage of gap in between the microbeam and the fixed electrode unless it does not trigger the instability by itself. Therefore, the instability that arises by domination of electric force over the mechanical force depends on both microbeam deflection and level of exciting voltage. Hence, if the magnitude of one factor is kept smaller, the other one must achieve larger values.

A comparison of diagrams shown in Figure 6 indicates that, compared to the instantaneous state, in durable state, smaller voltages can cause snap-through and pull-in instabilities. Therefore, at durable state which signifies viscoelastic behavior, two phenomena begin at less voltages than in instantaneous state, which represents purely elastic behavior. That is, the more is the gap for the viscoelastic 


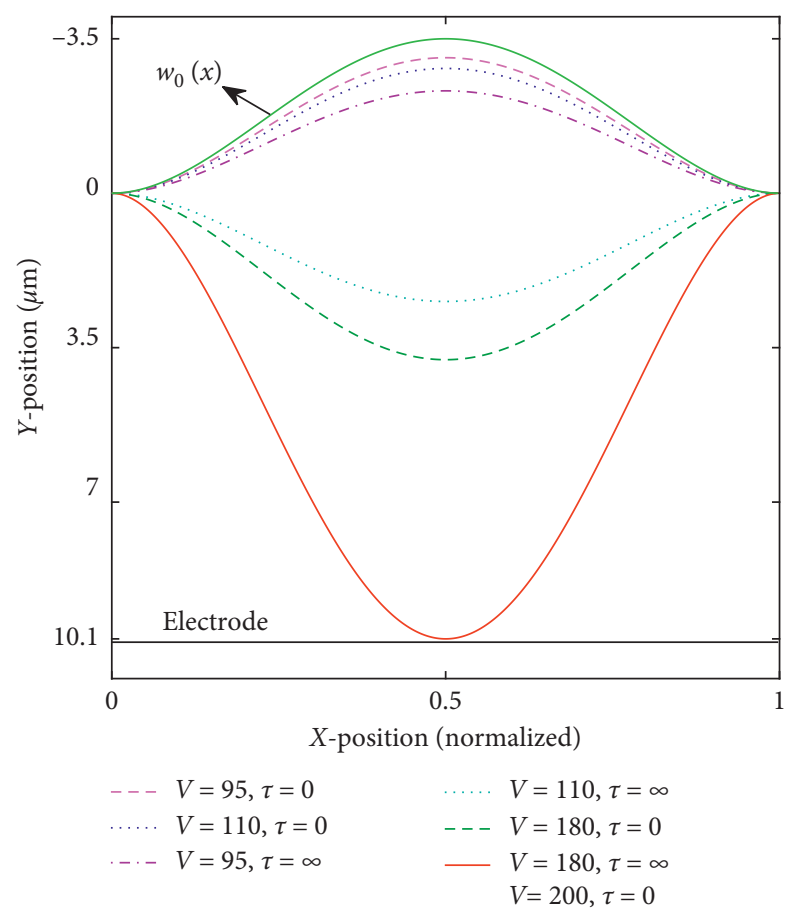

FIGURE 5: Instantaneous and durable position of microbeam at different actuation voltages.

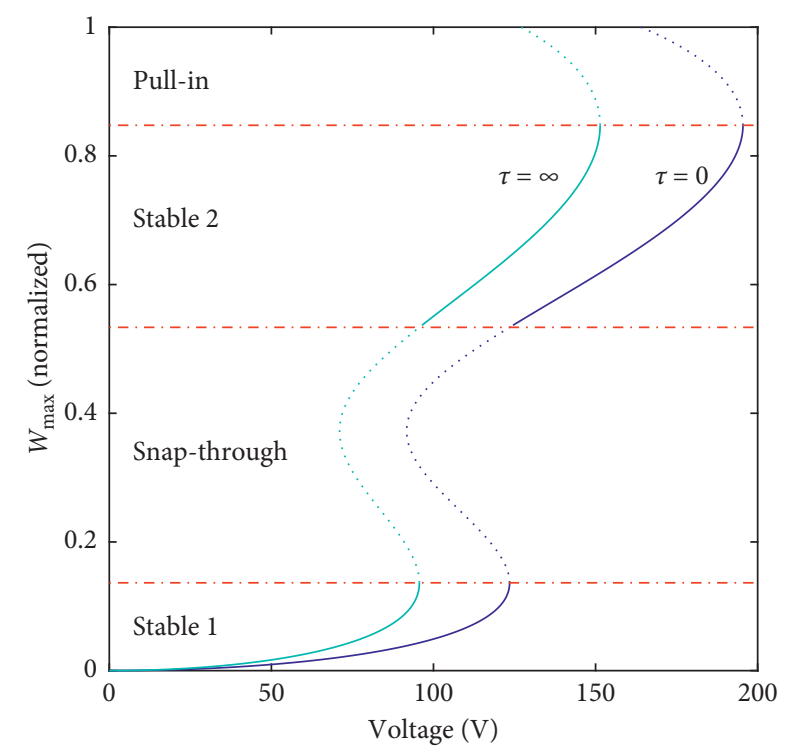

Figure 6: Maximum deflection versus voltage in the absence of axial load at $\tau=0$ and $\tau=\infty$.

structure to deform after actuation, the smaller would be the level of applied voltage which leads to the snap-through or pull-in instabilities. By using the deflection-voltage diagrams, the magnitude of position and voltage at the onset of these instantaneous and durable instabilities are obtained. For the case study without axial load, the characteristic instable deflections and voltages for the extreme states are shown in Table 3.

As Table 3 shows, in the absence of axial load, the beginning voltages of each one of these instabilities are different in the instantaneous and durable states but their positions are similar. These results indicate that threshold voltage tolerance of viscoelastic microbeam in extreme durable state is less than instantaneous state. Therefore, if deformation time trice is assigned between these two extreme states (a time between zero and infinity), the snapthrough occurs at a voltage between $95.6442 \mathrm{~V}$ and $123.4762 \mathrm{~V}$ and pull-in happens between $151.4068 \mathrm{~V}$ and $195.4654 \mathrm{~V}$. It means that, in smaller deformation periods, the deflection corresponding to a specific voltage would reduce and the microbeam stabilizes farther away from the fixed electrode. In this case, the magnitude of electric force reduces and would not suffice to overcome mechanical force needed for the structural instability. Thus, with less deformation opportunity, the system remains stable until higher levels of voltage are applied and the threshold voltage tolerance of the system is increased.

After determining the snap-through and pull-in voltages, in both instantaneous and durable responses, one can opine about the range of three different behaviors in Figure 5. Based on Figure 6, if applied voltage is selected between zero and $95.6442 \mathrm{~V}$ (less than durable snap-through voltage), the instantaneous response of the system specifies its initial stable state. In this voltage range, durable behavior only causes more displacement, so microbeam remains in its first stable configuration persistently. If actuation voltage is adjusted between $95.6442 \mathrm{~V}$ and $123.4762 \mathrm{~V}$ (between the instantaneous and durable snap-through voltages), the instantaneous response happens in its initial stable state, but durable behavior determines the microbeam position at the second stable configuration. In the case where the magnitude of applied voltage is between $123.4762 \mathrm{~V}$ and $151.4068 \mathrm{~V}$ (between the instantaneous snap-through voltage and the durable pull-in voltage), the instantaneous position indicates the second stable state and durable response also locates the second stable configuration, but with a higher displacement value. For actuation voltages between $151.4068 \mathrm{~V}$ and $195.4654 \mathrm{~V}$ (between instantaneous and durable pull-in voltages), the instantaneous behavior indicates the microbeam in the second stable condition, but the durable behavior causes unstable configuration. Obviously, if the magnitude of applied voltage is higher than $195.4654 \mathrm{~V}$ (instantaneous pull-in voltage), microbeam becomes immediately unstable and collapses onto the bottom electrode. According to the results, snap-through and pull-in voltages in two instantaneous and durable states are arranged similar to Figure 6. Depending on the voltage level, the status and shape of microbeam in two instantaneous and durable states are provided in Table 4.

Table 4 shows that considering more relaxation time for the viscoelastic curved microbeam would result in more deflection of microbeam. This effect in turn can cause a transition from the first stable position to the second stable state which is called snap-through phenomenon and in turn inspires a pull-in type of instability. It is considerable that such a categorization depends on the system dimensions and properties which determine the arrangement of the snapthrough and pull-in voltages of the instantaneous and durable states. Otherwise, it is possible to arrange instability 
TABLE 3: The characteristics of the instantaneous and durable instabilities in absence of axial load.

\begin{tabular}{lcccc}
\hline & \multicolumn{2}{c}{ Snap-through } & \multicolumn{2}{c}{ Pull-in } \\
& Deflection (normalized) & Voltage (V) & Deflection (normalized) & Voltage (V) \\
\hline Instantaneous & 0.1365 & 123.4762 & 0.8475 & 195.4654 \\
Durable & 0.1365 & 95.6442 & 0.8475 & 151.4068 \\
\hline
\end{tabular}

TABLE 4: The configuration of curved microbeam in instantaneous and durable states.

\begin{tabular}{lll}
\hline Voltage range* $^{\text {Instantaneous }}$ & \multicolumn{2}{c}{ Durable } \\
Position & Shape & Position \\
\hline $0<V<V_{S}^{D}$ & Stable 1 & Shape \\
$V_{S}^{D}<V<V_{P}^{I}$ & Stable 1 \\
$V_{S}^{I}<V<V_{P}^{D}$ & Stable 2 \\
$V_{P}^{D}<V<V_{P}^{I}$ & Stable 2 & Stable 2 \\
\hline
\end{tabular}

${ }^{*}$ D: durable, I: instantaneous, S: snap-through, and P: pull-in.

voltages in different situations such that effect of durable behavior on microbeam configuration is different from the conditions represented in Table 4.

In the following, the effects of several parameters upon the structural behavior of an exemplary microbeam in which its specifications are expounded in Table 2 are represented.

3.1. Effect of Actuation Voltage on the Stability of Durable Response. As mentioned in the beginning of this section, instantaneous behavior is calculated by taking $\tau=0$ and durable response is designated by $\tau \longrightarrow \infty$ in which $\tau$ is the relaxation time trice. Therefore, if structure relaxation time is between zero and infinity, the deflection of microbeam is also between instantaneous and durable states. To illustrate this condition, the variation of maximum deflection in between the instantaneous and durable states is obtained in different voltages. Figure 7 shows how the maximum microbeam deflection varies over the time for four different magnitudes of voltage levels.

In this case study the voltages are selected such that all four categories of Table 4 can be considered. Figure 7 and Table 4 show that relative magnitude of applied voltage (comparing instantaneous and durable instability voltages) determines the condition of microbeam transition from its instantaneous position to its durable state. This figure also confirms that, depending on relaxation time, one can determine the position and configuration of the microbeam. Moreover, based on this figure and the voltage level in the first row of Table 4, for $95 \mathrm{~V}$ excitation voltage by passing the time and approaching the stable response only the deflection of microbeam in its first stable position is increased.

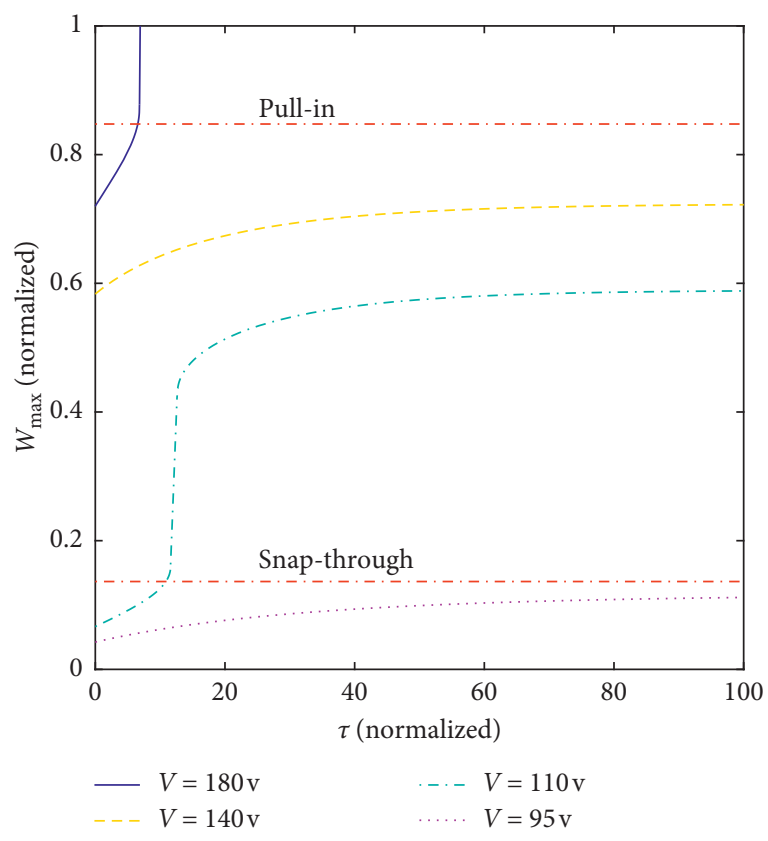

Figure 7: Different types of time histories for the midpoint deflection of microbeam.

As an example, for an applied voltage of $110 \mathrm{~V}$, if the relaxation time is close to $\tau=11$ units, that is, $t \longrightarrow 11 \times T$ ( $T$ is defined in equation (6)), microbeam can experience more deflection in its first stable state. But, if the passed time is more than this period, snap-through occurs and transfers the beam onto its second stable configuration. Yet with more relaxation time, in this state the displacement would increase without forming any new unstable condition. After this stage, having longer relaxation times, the microbeam position increases gradually with reducing deformation rate until a time period of about 80 unites in which it would reach a stable or durable state.

The excitation voltage of $140 \mathrm{~V}$ belongs to the third group of voltage levels in Table 4. Combined results of Figure 7 and Table 4 confirm that, in this voltage, microbeam excitation is in its second stable mode from the beginning and, by passing the time, it remains in this stable condition, but its deflection is increased.

As the fourth row in Table 4 recommends, the results shown in Figure 7 confirm that finally the microbeam becomes unstable and jumps into its second stable condition. In this case of $180 \mathrm{~V}$ excitation voltage, as the release time approaches 7 units, the microbeam loses its stability and pulls-into the electrode. 


\subsection{Effect of Actuation Voltage on Characteristics of Stable} Position. The deformation from instantaneous state to durable state is due to the time-dependent deformation of viscoelastic material at constant force. Since electrical force is generated by potential difference, the voltage level determines the necessary time to achieve durable response. In order to illustrate this behavior, the midpoint position of microbeam between two instantaneous and durable states is obtained for different voltage levels. The applied voltages are selected so that response of all studied cases matches a similar state in Table 4. In this case, Figure 8 shows the obtained results belong to the situation in which the studied beam loaded such that no snap-through or pull-in effect can be aroused.

The graphs in Figure 8 confirm that for all available modes whose excitation voltages match forth line in Table 4, permitting longer deformation period only causes more deflection in the second stable mode of microbeam. In fact, each one of the graphs in Figure 8 has two important characteristics. One feature is the final position of microbeam and the other one is the approximate period needed to achieve this position. The comparison of the diagrams in Figure 8 reveals that the more is the applied voltage, the more would be the attractive electrical force applied into the beam and consequently the durable position of the beam. Besides, it is seen that, in similar voltage differences, the larger is the voltage level, the larger would be the difference in between the durable responses. For example, the difference between the durable responses at $145 \mathrm{~V}$ and $150 \mathrm{~V}$ (i.e., $0.81-0.76=0.05)$ is more than twice the difference between the responses of $125 \mathrm{~V}$ and $130 \mathrm{~V}$ (i.e., $0.67-0.65=0.02$ ), while, in both cases, the difference between the voltages is $5 \mathrm{~V}$. Therefore, at higher excitation voltages, the effect of viscoelastic behavior and consequently the size of deflection to reach durable state is greater.

The comparison of the graphs in Figure 8 also shows that higher applied voltage levels result in more relaxation time trices. That is, the larger is the excitation voltage, the longer would be the period that microbeam takes to reach its durable position which follows the instantaneous excitation. This feature is due to the greater influence of viscoelasticity and greater distance between instantaneous and durable positions at higher excitation voltages. Since higher voltage means greater force, down to viscoelastic behavior of material, the higher is the excitation force, the larger would be the timedepended strains. These charts also show that the closer is the microbeam to its durable position, the less would be the displacement caused by its viscoelastic behavior. It means that, the main difference in between the instantaneous and durable positions is produced at the initial release intervals.

\subsection{Effect of Creep Modulus on Microbeam Relaxation} Response. Apparently, viscoelasticity commands the behavior of microbeam in instantaneous and durable states. This is investigated by determining the variation of microbeam position at different creep moduli (B) between two extreme instantaneous and durable states at $140 \mathrm{~V}$ voltage level. Figure 9 shows the results for microbeam position between these two states.

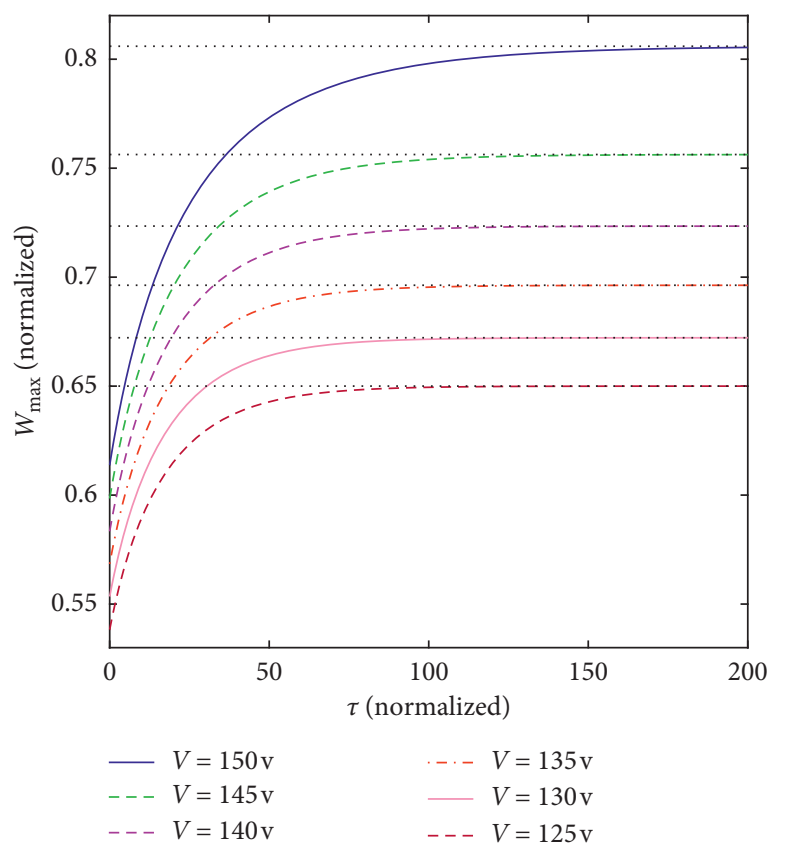

Figure 8: Time history of microbeam maximum deflection in second stable configuration.

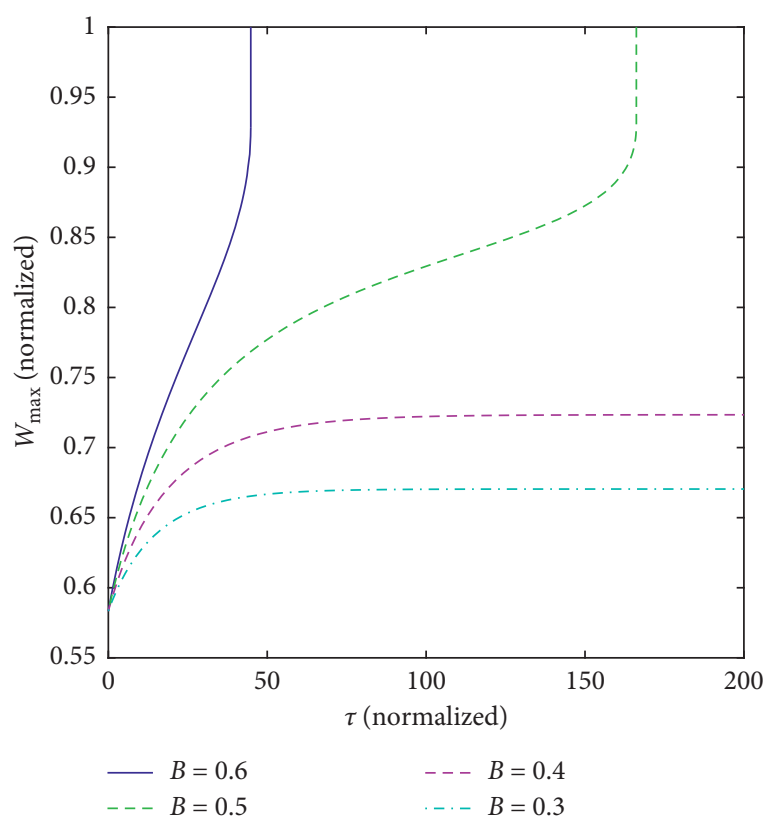

FIGURE 9: Maximum deflection between instantaneous and durable states at different creep moduli.

Higher viscosity ratio causes more deformation in constant stress. Therefore, a viscoelastic microbeam made of a material with bigger creep modulus shows bigger deformation level during relaxation time.

The curves in Figure 9 also show that, despite the same instantaneous position for the samples with different viscoelastic coefficients, the larger is this coefficient (i.e., the greater is the share of viscous behavior), the larger would be the durable position of microbeam. On the other hand, 
according to Figure 6, if microbeam reaches a certain position, it would dismiss its stable state. Therefore, as shown in Figure 9, among the microbeams in which their durable response would lead to the instable condition, the beam with higher viscosity coefficient achieves the unstable position in shorter period and thus collapses onto the fixed electrode more easily. For example, when creep modulus equals 0.6 , the microbeam collapses after nearly 44 time-units while a microbeam with creep modulus of 0.5 collapses after 166 time-units. The comparison of structure behavior in creep modulus between 0.3 and 0.4 shows that larger creep modulus leads to more relaxation strains. Therefore, although the instantaneous position of both samples equals 0.5834 units, the 0.3 -creep modulus leads to a durable position of 0.6704 units and the 0.4 -creep modulus leads to a durable position of 0.7234 units.

Therefore, among the microbeams which are stable in durable state, the beams with greater creep modulus merit larger durable positions and achieve their durable state in larger periods.

\subsection{Effect of Relaxation Coefficient on Microbeam Relaxed} State. Equation (2) shows that relaxation coefficient $\lambda$ affects the necessary time trice needed for the achievement of a certain position for the viscoelastic microbeam. In order to investigate the effect of this parameter, the displacement of microbeam between two instantaneous and durable states is extracted for different values of this coefficient. Figure 10 shows the results for an excitation voltage of $140 \mathrm{~V}$.

Figure 10 shows that coefficient $\lambda$ has no effect on the size of durable position. It means that, for the same applied voltages, despite the level of relaxation coefficient, the durable positions will be the same, even though the necessary time to reach this position is not the same. In fact, the larger is the relaxation coefficient the sooner is the incursion of durable position. Figure 10 is obtained for the condition where microbeam remains stable in durable state. Similarly, the variation of maximum deflection is extracted in between the two instantaneous and durable states in which system remains stable. Figure 11 shows the result for four different values of relaxation coefficient in a voltage of $170 \mathrm{~V}$.

Similar to Figure 6, Figure 11 also shows that in the absence of axial load, the instability always occurs at the same position. Therefore, the smaller is the coefficient $\lambda$, the more would be the time needed to achieve pull-in position. It means that instability commences at a longer period. If applied voltage leads to an unstable durable response (here, in voltage levels between $151.4068 \mathrm{~V}$ and $195.4654 \mathrm{~V}$ ), pull-in instability occurs at larger levels of relaxation coefficient $\lambda$ at shorter periods. The effect of this relaxation coefficient upon the snap-through is similar to its effect on the pull-in type of instability. That is, the higher is the relaxation coefficient, the faster is the happening of snap-through instability to transfer the microbeam into a second stable configuration.

As a whole, the results show that the characteristics of snap-through and pull-in instabilities in durable state, as representative of viscoelastic behavior, depend on various parameters such as the creep modulus $B$ and relaxation

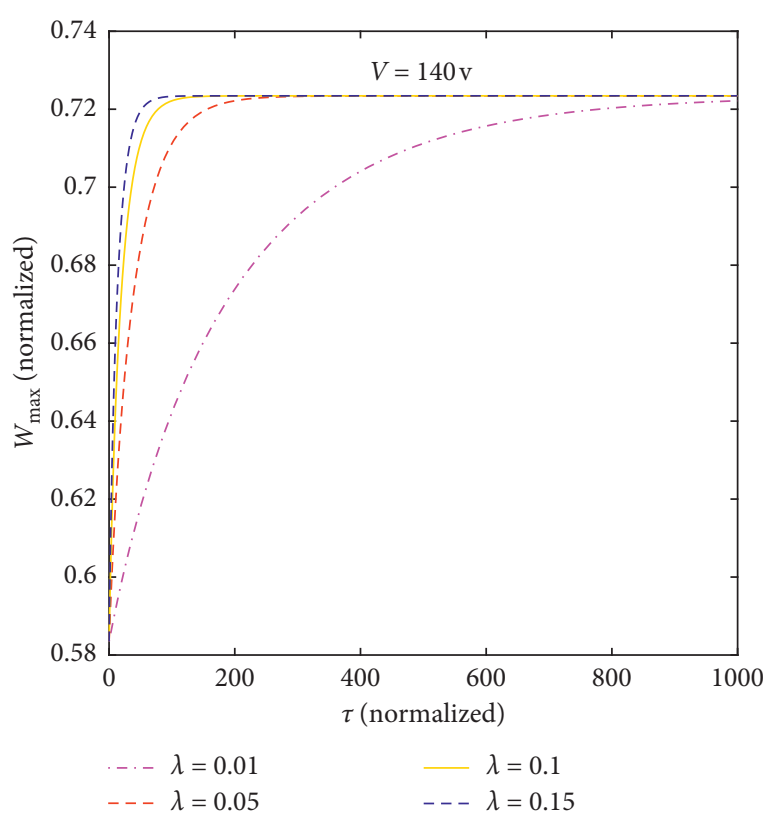

Figure 10: Maximum deflection between instantaneous and durable states for different values of relaxation coefficient.

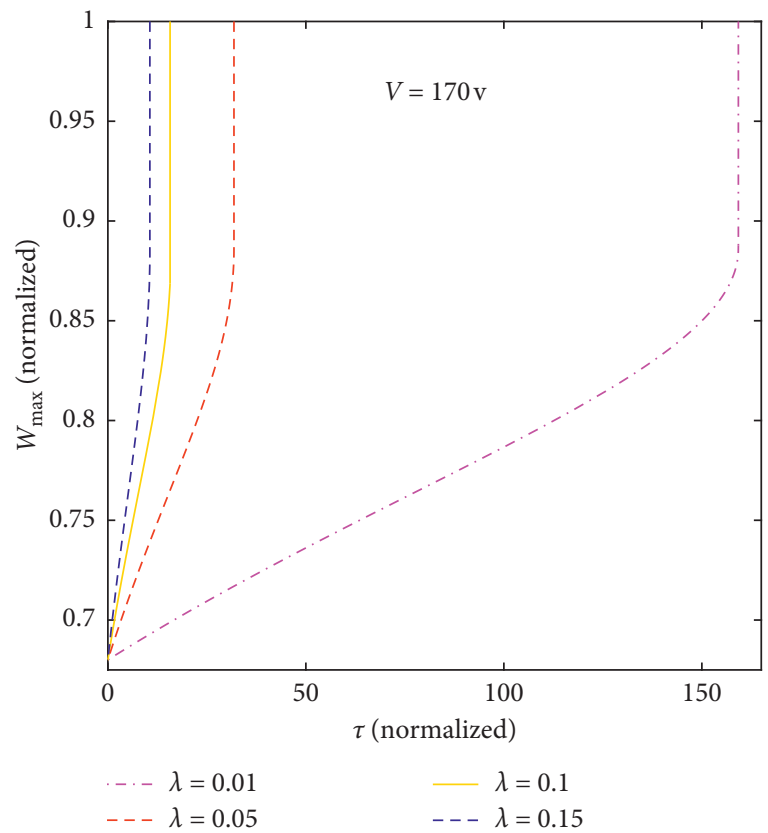

FIGURE 11: Maximum deflection between instantaneous response and pull-in at different values of relaxation coefficient.

coefficient $\lambda$. In other words, 6-11 show the instabilities introduced by the characteristic parameters of location, voltage, and time-delay. Figure 12 simultaneously shows the durable unstable position versus these two coefficients.

Figure 12 confirms the previous discussion that, in the absence of axial load, snap-through and pull-in locations do not depend on creep modulus and relaxation coefficients. Hence, in the represented case study, despite the magnitude of viscoelastic and relaxation coefficients, snap-through 


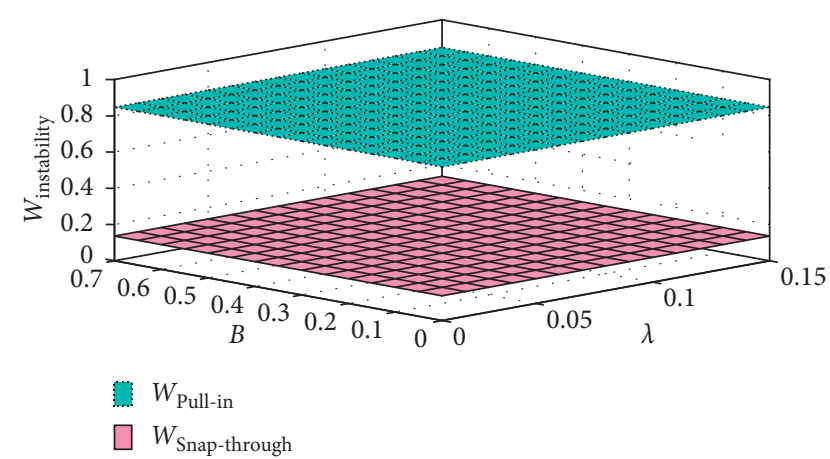

FIGURE 12: Snap-through and pull-in positions in durable state versus the creep modulus and relaxation coefficients.

happens whenever nondimensional position receives 0.1365 units and pull-in happens when nondimensional position equals 0.8475 units. Similarly, the effects of these parameters on the size of instability voltages are also investigated in durable state as representatives of viscoelastic behavior. Figure 13 shows the variation of snap-through and pull-in voltages in durable state, versus the creep modulus and relaxation coefficient.

Figure 13 shows that relaxation coefficient does not affect the size of instability voltages. However, as the viscosity ratio of material increases, the size of these voltages will decrease conversely. In this figure, the zero-creep modulus signifies the elastic material. Therefore, according to Figure 13, in durable state and larger levels of creep moduli $(B \longrightarrow 1)$, smaller actuation voltages may trigger snap-through or pullin instabilities more easily. Also, in this case, the voltage difference in between these two unstable states would decrease accordingly. Besides, it shows that a pull-in instability which follows the snap-through effect would happen in less level of voltage differences.

Based on Figures 12 and 13 in the absence of axial load, snap-through and pull-in positions are not dependent on the viscoelastic behavior rather they are equal to their position obtained by pure elastic model. Nonetheless, the voltage magnitude of these phenomena in durable state is depended on the material behavior. These results are obtained considering the durable state as the representative of viscoelastic behavior. Considering durable state means that instability criteria are only assessed in long-term periods and the instability is not investigated in short-term durations. In this situation, the system behavior is considered to be time-independent. Therefore, the effect of relaxation coefficient would be neglected. But the nature of viscous behavior and the results in Figure 5 predict that threshold voltage tolerance of the system is depended on the period of deformation. In other words, if, in place of durable or steady assumption for the beam deformation, one reflects back to more general transient type of deformation, the effect of relaxation coefficient is revealed. To illustrate this effect, the magnitudes of snap-through and pull-in voltages are obtained by limiting the time trice to 1000 units, in different sizes of creep modulus and relaxation coefficients. Figures 14 and 15 show the snap-through and pull-in voltages, considering this time limitation.

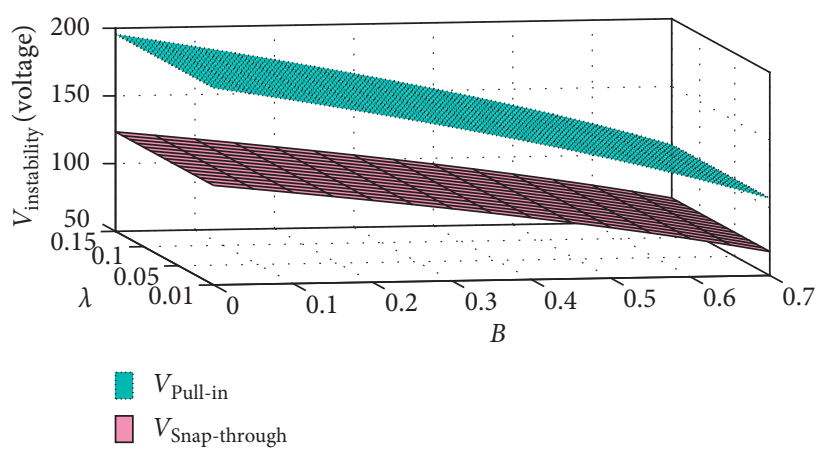

FIgURE 13: Snap-through and pull-in voltages in durable state versus the creep modulus and relaxation coefficients.

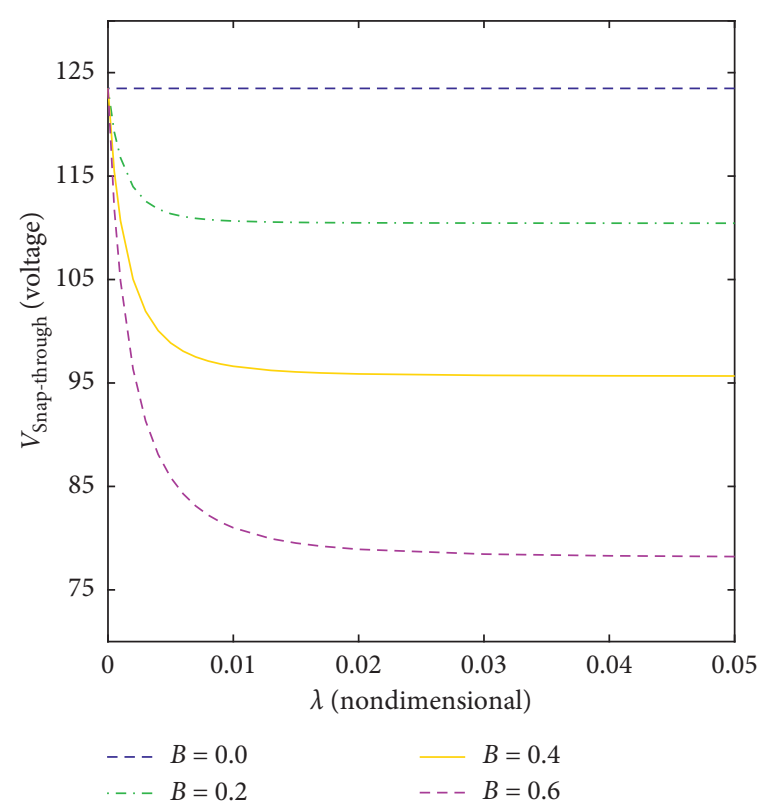

Figure 14: Dependence of snap-through voltage on relaxation coefficient for different amounts of creep moduli.

Figures 10 and 11 have shown that larger relaxation coefficient causes greater movement at a definite time trice. So, as Figure 14 shows, the microbeam reaches snap-through position at a smaller voltage level and moves earlier into its second stable position. Figure 14 also shows that most decline in viscoelastic snap-through voltage appears in small levels of relaxation coefficients. In fact, the studied period is large enough to let the structure approaches its durable position in smaller amounts of $\lambda$. In Figure 9, it is shown that larger viscoelastic coefficient results in higher amounts of deflection at definite periods. Thus, in larger amounts of this factor $(B)$, microbeam reaches snap-through position at lower voltage levels. In other words, as shown in Figure 14, the size of snap-through voltage decreases by increasing the contribution of material viscosity. Similarly, Figure 15 shows the variation of pull-in voltage at a limited time trice, for different values of viscoelastic and relaxation coefficients.

Additionally, based on Figure 15 and close similar to the snap-through voltage effect, the major part of variation in pull-in voltage occurs in small values of relaxation 


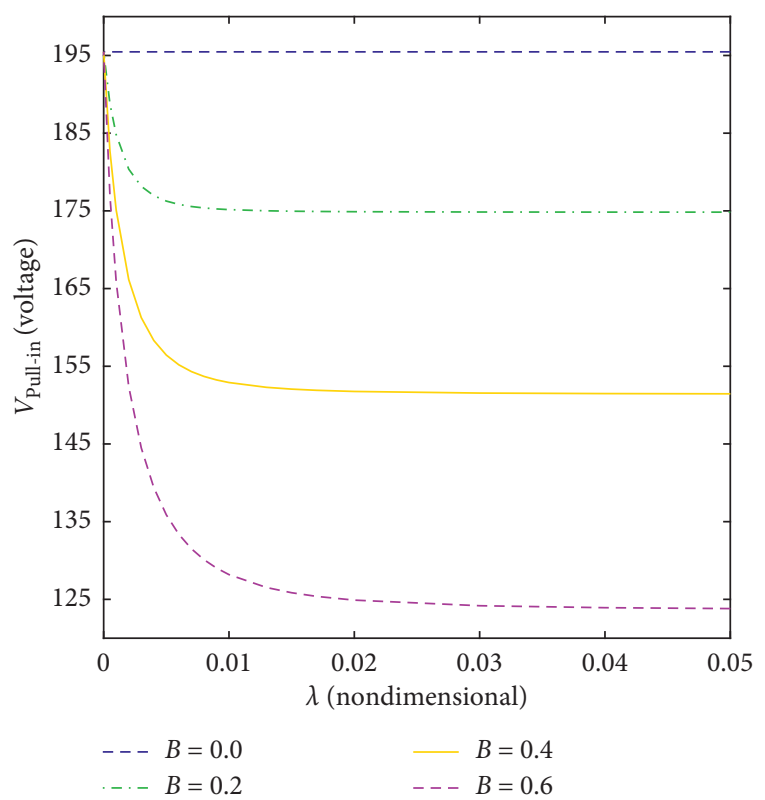

FIGURE 15: Dependence of pull-in voltage on relaxation coefficient in different viscoelastic coefficients.

coefficient. Oppositely, in large values of this coefficient, the magnitude of pull-in voltage is approximately equal to its durable value. Therefore, selecting larger coefficients will not assure more change on microbeam position at this limited time trice period. As a result, one can say that the effect of viscoelastic behavior, especially in larger amounts of relaxation coefficient and longer relaxation periods, would be appreciable.

\section{Conclusions}

The effects of viscoelasticity on snap-through and pull-in types of microbeam instantaneous and durable instabilities are studied. The results show that, in comparison with purely elastic microbeams, viscoelasticity would reduce durable snap-through and pull-in voltages. At higher amounts of creep modulus $B$, the difference between snap-through and pull-in voltages is reduced. Once the study is limited to a definite time period, considering larger relaxation coefficients $(\lambda)$ results in reduction of threshold instability voltages, significantly. In that case, the reduction is severe in smaller amounts of the coefficient, while in larger amounts of $\lambda$, the magnitude of voltage is approximately equal to its corresponding amount at durable state. Furthermore, the results show that, in the absence of axial load, unstable positions of elastic and viscoelastic microbeams are the same. The viscoelastic and relaxation coefficients determine the magnitude of deflection at any time and voltage level. Based on this study, the larger is the amounts of these coefficients, the shorter would be the time trice to shape durable position of microbeam and the less would be the amount of threshold voltage which unbalances the microbeam. Therefore, the rate of voltage which determines the rate of deformation would also affect the extent of instable parameters including the position, voltage, and time characteristic parameters.

\section{Appendix}

$$
\begin{aligned}
a_{i} & =\int_{0}^{1} \psi^{i}(x) \mathrm{d} x, \quad i=1,2,3,4, \\
b_{i} & =\int_{0}^{1} \psi^{i}(x) \frac{\mathrm{d}^{2} \psi(x)}{\mathrm{d} x^{2}} \mathrm{~d} x, \quad i=1,2,3, \\
c_{i} & =\int_{0}^{1} \psi^{i}(x) \frac{\mathrm{d}^{4} \psi(x)}{\mathrm{d} x^{4}} \mathrm{~d} x, \quad i=1,2,3, \\
d_{i} & =\int_{0}^{1} \psi^{i}(x) \frac{\mathrm{d}^{2} w_{0}(x)}{\mathrm{d} x^{2}} \mathrm{~d} x, \quad i=1,2,3, \\
e_{i} & =\int_{0}^{1} w_{0}(x) \psi^{i}(x) \frac{\mathrm{d}^{2} \psi(x)}{\mathrm{d} x^{2}} \mathrm{~d} x, \quad i=1,2, \\
f_{i} & =\int_{0}^{1} w_{0}(x) \psi^{i}(x) \frac{\mathrm{d}^{4} \psi(x)}{\mathrm{d} x^{4}} \mathrm{~d} x, \quad i=1,2, \\
m_{i} & =\int_{0}^{1} w_{0}(x) \psi^{3}(x) \mathrm{d} x, \\
g & =\int_{0}^{1} w_{0}^{2}(x) \psi(x) \frac{\mathrm{d}^{4} \psi(x)}{\mathrm{d} x^{4}} \mathrm{~d} x, \\
h_{i} & =\int_{0}^{1} w_{0}^{i}(x) \psi^{2}(x) \mathrm{d} x, \quad i=1,2,
\end{aligned}
$$

\section{Data Availability}

The data used to support the findings of this study are available from the corresponding author upon request.

\section{Disclosure}

This paper is the result of academic research required for the fulfilment of the graduation conditions of Ph.D. students at the Ferdowsi University of Mashhad in Iran. It is a purely theoretical attempt and has not absorbed extra funding.

\section{Conflicts of Interest}

The authors declare that there are no conflicts of interest regarding the publication of this paper.

\section{References}

[1] N. Choudhary and D. Kaur, "Vibration damping materials and their applications in nano/micro-electro-mechanical systems: a review," Journal of Nanoscience and Nanotechnology, vol. 15, no. 3, pp. 1907-1924, 2015. 
[2] W. H. Ko, "Trends and frontiers of MEMS," Sensors and Actuators A: Physical, vol. 136, no. 1, pp. 62-67, 2007.

[3] G. Hu and W. Liu, "Nano/micro-electro mechanical systems: a patent view," Journal of Nanoparticle Research, vol. 17, no. 12, p. $465,2015$.

[4] M. I. Younis, "Microbeams," in MEMS Linear and Nonlinear Statics and Dynamics, pp. 251-357, Springer, Boston, MA, USA, 2011.

[5] J. Zhang and Y. Fu, "Pull-in analysis of electrically actuated viscoelastic microbeams based on a modified couple stress theory," Meccanica, vol. 47, no. 7, pp. 1649-1658, 2012.

[6] G. I. Taylor, "The coalescence of closely spaced drops when they are at different electric potentials," Proceedings of the Royal Society of London. Series A. Mathematical and Physical Sciences, vol. 306, pp. 423-434, 1968.

[7] H. C. Nathanson, W. E. Newell, R. A. Wickstrom, and J. R. Davis, "The resonant gate transistor," IEEE Transactions on Electron Devices, vol. 14, no. 3, pp. 117-133, 1967.

[8] H. M. Ouakad and M. I. Younis, "The dynamic behavior of MEMS arch resonators actuated electrically," International Journal of Non-linear Mechanics, vol. 45, no. 7, pp. 704-713, 2010.

[9] J. Qiu, J. Lang, and A. Slocum, “A centrally-clamped parallelbeam bistable MEMS mechanism," in Proceedings of the 14th IEEE International Conference on MEMS, pp. 353-356, Interlaken, Switzerland, January 2001.

[10] L. Zhang, L. N. Zhang, M. Y. Wang, G. Q. Li, and Z. T. Sui, "Recovery of titanium compounds from molten Ti-bearing blast furnace slag under the dynamic oxidation condition," Minerals Engineering, vol. 20, no. 7, pp. 684-693, 2007.

[11] Y. Zhang, Y. Wang, and Z. Li, "Analytical method of predicating the instabilities of a micro arch-shaped beam under electrostatic loading," Microsystem Technologies, vol. 16, no. 6, pp. 909-918, 2010.

[12] M. M. Zand, "The dynamic pull-in instability and snapthrough behavior of initially curved microbeams," Mechanics of Advanced Materials and Structures, vol. 19, no. 6, pp. 485-491, 2012.

[13] M. I. Younis, H. M. Ouakad, F. M. Alsaleem, R. Miles, and W. Cui, "Nonlinear dynamics of MEMS arches under harmonic electrostatic actuation," Journal of Microelectromechanical Systems, vol. 19, no. 3, pp. 647-656, 2010.

[14] K. Bethe, D. Baumgarten, and J. Frank, "Creep of sensor's elastic elements: metals versus non-metals," Sensors and Actuators A: Physical, vol. 23, no. 1-3, pp. 844-849, 1990.

[15] M. Elwenspoek and H. Jansen, Silicon Micromachining, Cambridge University Press, Cambridge, UK, 2004.

[16] S. Schmid, P. Senn, and C. Hierold, "Electrostatically actuated nonconductive polymer microresonators in gaseous and aqueous environment," Sensors and Actuators A: Physical, vol. 145-146, pp. 442-448, 2008.

[17] X. Yan, W. L. Brown, Y. Li et al., "Anelastic stress relaxation in gold films and its impact on restoring forces in MEMS devices," Journal of Microelectromechanical Systems, vol. 18, no. 3, pp. 570-576, 2009.

[18] K. Tuck, A. Jungen, A. Geisberger, M. Ellis, and G. Skidmore, "A study of creep in polysilicon MEMS devices," Journal of Engineering Materials and Technology, vol. 127, no. 1, pp. 90-96, 2005.

[19] K. P. Larsen, A. A. Rasmussen, J. T. Ravnkilde, M. Ginnerup, and O. Hansen, "MEMS device for bending test: measurements of fatigue and creep of electroplated nickel," Sensors and Actuators A: Physical, vol. 103, no. 1-2, pp. 156-164, 2003.
[20] H.-J. Lee, P. Zhang, and J. C. Bravman, "Stress relaxation in free-standing aluminum beams," Thin Solid Films, vol. 476, no. 1, pp. 118-124, 2005.

[21] Y. M. Fu and J. Zhang, "Nonlinear static and dynamic responses of an electrically actuated viscoelastic microbeam," Acta Mechanica Sinica, vol. 25, no. 2, pp. 211-218, 2009.

[22] M. H. Ghayesh, H. Farokhi, and S. Hussain, "Viscoelastically coupled size-dependent dynamics of microbeams," International Journal of Engineering Science, vol. 109, pp. 243-255, 2016.

[23] M. A. Attia and S. A. Mohamed, "Nonlinear modeling and analysis of electrically actuated viscoelastic microbeams based on the modified couple stress theory," Applied Mathematical Modelling, vol. 41, pp. 195-222, 2017.

[24] L. Li, Q. Zhang, W. Wang, and J. Han, "Dynamic analysis and design of electrically actuated viscoelastic microbeams considering the scale effect," International Journal of Non-linear Mechanics, vol. 90, pp. 21-31, 2017.

[25] A. H. Ramini, Q. M. Hennawi, and M. I. Younis, "Theoretical and experimental investigation of the nonlinear behavior of an electrostatically actuated in-plane MEMS arch," Journal of Microelectromechanical Systems, vol. 25, no. 3, pp. 570-578, 2016.

[26] A. R. Askari and M. Tahani, "An alternative reduced order model for electrically actuated micro-beams under mechanical shock," Mechanics Research Communications, vol. 57, pp. 34-39, 2014.

[27] R. C. Batra, M. Porfiri, and D. Spinello, "Vibrations of narrow microbeams predeformed by an electric field," Journal of Sound and Vibration, vol. 309, no. 3-5, pp. 600-612, 2008.

[28] Y. H. Qian, D. X. Ren, S. K. Lai, and S. M. Chen, "Analytical approximations to nonlinear vibration of an electrostatically actuated microbeam," Communications in Nonlinear Science and Numerical Simulation, vol. 17, no. 4, pp. 1947-1955, 2012.

[29] Y. Fu, J. Zhang, and L. Wan, "Application of the energy balance method to a nonlinear oscillator arising in the microelectromechanical system (MEMS)," Current Applied Physics, vol. 11, no. 3, pp. 482-485, 2011. 